\section{Science in Greece}

\section{Starting almost from scratch}

GREECE is about to take political notice of science - which means it may have a science policy, and a reasonable science budget, for the first time since the sack of Alexandria. According to the newlyappointed minister of research and technology, Professor George Lianis, the Greek government aims to multiply its research and development spending sixfold over the next five years - and to reform or create whatever institutions are necessary in the process.

Lianis knows about reform in Greece: he was one of a team of radical ministers appointed to the ministry of education by socialist Prime Minister Andreas Papandreou, when the PASOK party came to power in October 1981. That team attempted to bring the universities up to date, and in less than a year pushed through a bill which reduced the power of the previously autocratic professors, gave recognition and a career structure to other members of staff, established graduate schools and offered a degree of democratic power to the students.

Those major reforms are now working themselves through, and Lianis - an engineer trained at Imperial College, London - has turned to science. Melina Mercouri, the former actress, appointed minister of culture and science, will remain in that role, but her primary preoccupation will be the arts and archaeology.

Greek science is both "stifled by bureaucracy" and "anarchic" says Lianis, one of whose priorities will be to sort out which scientists in the universities and the few research institutions (such as the Hellenic Research Foundation and the Democritus nuclear research centre) are more or less political appointees of previous governments, and which are doing real science.

Then there must be proper structures for scientific assessment, and the allocation of grants. According to one Greek scientist on the telephone from Athens last week, "no-

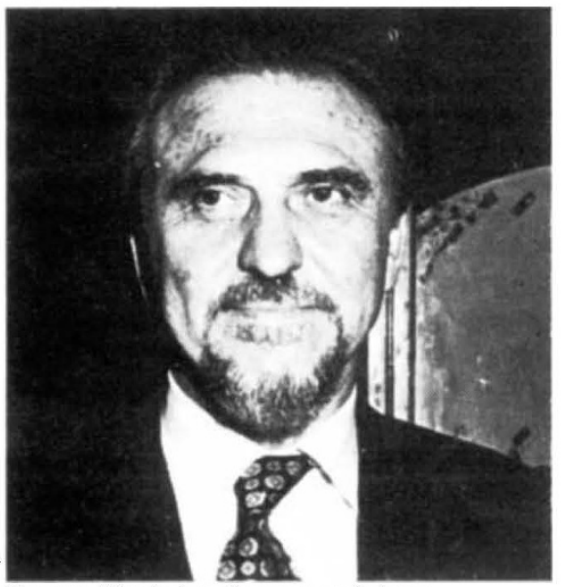

George Lianis heads a new ministry one has been properly reviewed before". For researchers outside the university reform, there is at present neither a career structure nor tenure, but a bill is being prepared to correct these failings.

Lianis hopes for much from expatriate Greek scientists - like himself - whom he expects will return out of a sense of duty, "nostalgia" for the home country, recognition and status, and an opportunity to create something new. "People will have to come and create the conditions for good research in Greece"' he says.

It is early to ask for policy, but Lianis stresses software and informatics, biotechnology, solar and geothermal energy, and the life sciences (for health and agriculture). $\mathrm{He}$ has been particularly impressed by a study which showed that Greece, with its many islands, fishing ports and warm waters, has tremendous potential for aquaculture, and he is seeking cooperation with Italy in this field.

Lianis's budget this year will be 3,000 million drachmas ( $£ 21$ million), including the salaries of his staff of 70 and the staff of the research institute - an apparently tiny sum, but in terms of equipping and establishing laboratories already double what was available last year. More cash is available to the universities and research institutes by direct funding of special projects through the treasury.

Lianis wishes to push the total Greek expenditure on research and development from 0.2 per cent of gross national product (1982) to 1.2 per cent (by 1987). Some of that, he hopes, will come from industry, which at present spends "next-tonothing".

\title{
Heavy ion research
}

\section{More machines come on-line}

THE French Grand Accelérateur National d'Ions Lourds (GANIL), a FF 557 million ( $£ 52.5$ million) heavy ion accelerator designed to probe a new energy region for nuclei, is officially opened this week by the French research minister. But GANIL has already produced its first remarkable results which throw a new light on the physics it will be surveying.

These results appear to show that nuclei "fragment" and behave like collections of individual particles at GANIL energies of around $45 \mathrm{MeV}$ per nucleon - a phenomenon hitherto believed to set in only at the much higher energies of $1,800 \mathrm{MeV}$ per nucleon used by the Bevalac heavy ion machine at Berkeley, California. If these early results of an Orsay group working at GANIL are confirmed, the "intermediate energy phenomena" which were to have been studied may not in fact exist.

The original argument went like this: GANIL would be aimed at an unexplored energy region between the $18 \mathrm{MeV}$ per nucleon available at GSI Darmstadt in West Germany (and at Dubna in the Soviet Union), and the $1,800 \mathrm{MeV}$ per nucleon available at the Bevalac machine. At the former energies, collisions yield high-mass composite nuclei, often at high states of spin; at the latter, nuclei appear to behave like gases of protons, neutrons and other nuclear fragments. In between, GANIL hoped - and hopes - to study a region where nuclei behave like collective nuclear "'matter", a material independent of particular nuclear structures or shapes but still composite: broadly speaking, the liquid state rather than the gas visible at Berkeley.

However, the first GANIL results appear far more like Berkeley measurements than expected: in particular, the projectile nucleus (argon-40 at $45 \mathrm{MeV}$ per nucleon) fragments on hitting its target into a spectrum of smaller nuclei (including phosphorus, chlorine and sulphur) which fly on with the same velocity as the projectile.

Meanwhile in Britain, two other accelerators have come on-line. One of them, the Nuclear Structure Facility, a 20 MV Van de Graaff electrostatic heavy ion accelerator at Daresbury, has produced its first results, and the other, the Spallation Neutron Source (for condensed matter studies) at the Rutherford and Appleton Laboratory at Didcot its first test beam.

At the Nuclear Structure Facility, a facility which works at lower energy than GANIL, and which cost $£ 13.6$ million and was eight years in construction, the first experiments - a collaboration between groups from the universities of Liverpool, Manchester and Copenhagen - have investigated high nuclear spin states using hafnium- 168 and titaniun- 48 beams, and discovered clear evidence for "spin depairing". Group spokesman John Lisle of the University of Manchester describes it thus: at low energies, the nucleons in nuclei have paired spins, and the nuclear material behaves rather like a superconductor (in which electrons pair up); but at high energies the nuclear spins appear to uncouple, producing something like a normal metal. Thus the normal state of nuclei appears like the abnormal (superconducting) state of metals, and the abnormal (excited) state of nuclei like the normal state of metals. Sketchy evidence for this was available a year ago, said Dr Lisle, but the Nuclear Structure Facility results show it clearly for the first time.

The Nuclear Structure Facility appears to have had this success not just because of the accelerator - a close equivalent at Oak Ridge National Laboratory in the United States has been running for a year, and another is ready in Japan - but because of a unique gamma-ray apparatus called TESSA, which was used to analyse the decay spectra of the spinning nuclei with great accuracy.
Robert Walgate 